\title{
A RIBBON KNOT GROUP WHICH HAS NO FREE BASE
}

\section{KATSUYUKI YOSHIKAWA}

(Communicated by Haynes R. Miller)

\begin{abstract}
We consider the following problem: If a group $G$ satisfies the conditions (1) $G$ has a finite presentation with $r+1$ generators and $r$ relators, and (2) there exists an element $x$ of $G$ such that $G=\langle\langle x\rangle\rangle^{G}$ where $\langle\langle x\rangle\rangle^{G}$ is the normal closure of $x$ in $G$, then is $G$ an HNN (Higman-Neumann-Neumann) extension of a free group of finite rank? In this paper, we give a negative answer to the problem. Thus it follows that there exists a ribbon $n$-knot group $(n \geq 2)$ which has no free base.
\end{abstract}

1. Introduction. Let $G$ be a 2-knot group, i.e., the group of some 2-sphere smoothly embedded in the 4 -sphere $S^{4}$. Then it is easily seen that if $G$ is an HNN extension of some free group of finite rank, then $G$ has deficiency one. Conversely, the following conjecture is raised:

CONJECTURE. If the deficiency of $G$ is one, then $G$ has a free base of finite rank.

The purpose of this paper is to give counterexamples to this conjecture, that is,

THEOREM 1. Let $G$ be a group presented by

$$
\left\langle a, b, t: a^{p}=b^{q}, t a^{\alpha} t^{-1}=b^{\beta}\right\rangle
$$

where $p, q, \alpha, \beta$ are nonzero integers such that $p \beta-q \alpha= \pm 1$ and $p, q, \alpha, \beta \neq \pm 1$. Then,

(1) $G$ is a ribbon 2-knot group, i.e., a 2-knot group with Wirtinger presentation of deficiency one, and

(2) $G$ has no free base of finite rank.

Thus, our examples show that Gutierrez's theorem [2, p. 287, Theorem (iii)] is false. In $\S 4$, we prove that any 2-knot group with one-relator Wirtinger presentation has a free base of finite rank.

2. Preliminaries. Let $\left\{A_{i}, \theta_{j k}\right\}$ be a collection of groups $A_{i}$ and isomorphisms $\theta_{j k}: U_{j k} \rightarrow U_{k j}$ associated with certain pairs of $A_{j}, A_{k}$ such that $\theta_{j k}=\theta_{k j}^{-1}$, where $U_{j k}$ and $U_{k j}$ are subgroups of $A_{j}$ and $A_{k}$ respectively. With the collection $\left\{A_{i}, \theta_{j k}\right\}$ we associate a linear graph each of whose vertices corresponds to a group $A_{i}$ and each of whose edges joins two vertices $A_{j}$ and $A_{k}$ if there exists $\theta_{j k}$ (and hence $\theta_{k j}$ ).

Received by the editors October 29, 1985.

1980 Mathematics Subject Classification (1985 Revision). Primary 57Q45; Secondary 20E06, $20 \mathrm{~F} 05$.

Key words and phrases. Knot group, HNN extension. 
If this graph is a tree, the group defined by

$$
\left\langle\prod_{i} * A_{i}: U_{j k}=\theta_{j k}\left(U_{j k}\right) \text { for all edges } e_{j k}\right\rangle
$$

is called a tree product of the factors $A_{i}$ (with the subgroups $U_{j k}$ and $U_{k j}$ amalgamated under $\theta_{j k}$ ) [4].

An HNN extension with more than one stable letter is defined as follows [4, 5]: Let $H$ be a group and let $\left\{A_{1}, \ldots, A_{n}\right\},\left\{B_{1}, \ldots, B_{n}\right\}$ be sets of subgroups of $H$ with isomorphisms $\phi_{i}: A_{i} \rightarrow B_{i}, i=1, \ldots, n$. Then the $H N N$ extension of the base $H$ with stable letters $t_{1}, \ldots, t_{n}$ and associated subgroups $\left\{A_{1}, \ldots, A_{n}\right\}$ and $\left\{B_{1}, \ldots, B_{n}\right\}$ is the group given by

$$
\left\langle H, t_{1}, \ldots, t_{n}: t_{i} a_{i} t_{i}^{-1}=\phi_{i}\left(a_{i}\right), a_{i} \in A_{i}, i=1, \ldots, n\right\rangle .
$$

Finally, we give some lemmas. Let $G$ be as in Theorem 1 , and let $H=g p\{a, b\}=$ $\left\langle a, b: a^{p}=b^{q}\right\rangle$.

LEMMA 2.1. The element $b$ is not conjugate to $a^{\alpha k}$ or $b^{\beta k}$ in $H$ for any integer k. Similarly, $a$ is not conjugate to $a^{\alpha k}$ or $b^{\beta k}$.

PROOF. Let $f$ be the homomorphism of $H$ onto the infinite cyclic group $Z=\langle z:\rangle$ defined by $f(a)=z^{q}$ and $f(b)=z^{p}$. Then, since $p \beta-q \alpha= \pm 1$ and $p, q, \alpha, \beta \neq \pm 1$, it follows that $f\left(a^{\alpha k}\right)$ and $f\left(b^{\beta k}\right)$ are different from $z^{p}$ and $z^{q}$. Therefore the lemma holds.

LEMMA 2.2. For any $S \in G$, a does not commute $S b S^{-1}$ in $G$.

PROOF. Let $S=g_{0} t^{\varepsilon_{1}} g_{1} \cdots t^{\varepsilon_{n}} g_{n}$ be reduced, that is, $\varepsilon_{i}= \pm 1, g_{i} \in H$, and there is no subword $t g_{i} t^{-1}$ with $g_{i} \in g p\left\{a^{\alpha}\right\}$ or $t^{-1} g_{i} t$ with $g_{i} \in g p\left\{b^{\beta}\right\}$ (cf. [5]). Then, we have

$$
\begin{aligned}
& a\left(S b S^{-1}\right) a^{-1}\left(S b^{-1} S^{-1}\right) \\
& =a\left(g_{0} t^{\varepsilon_{1}} \cdots t^{\varepsilon_{n}} g_{n}\right) b\left(g_{0} t^{\varepsilon_{1}} \cdots t^{\varepsilon_{n}} g_{n}\right)^{-1} \\
& \quad \cdot a^{-1}\left(g_{0} t^{\varepsilon_{1}} \cdots t^{\varepsilon_{n}} g_{n}\right) b^{-1}\left(g_{0} t^{\varepsilon_{1}} \cdots t^{\varepsilon_{n}} g_{n}\right)^{-1} .
\end{aligned}
$$

By Lemma 2.1, we can see that

$$
g_{n} b^{ \pm 1} g_{n}^{-1} \notin g p\left\{a^{\alpha}\right\} \cup g p\left\{b^{\beta}\right\},
$$

and

$$
g_{0}^{-1} a^{-1} g_{0} \notin g p\left\{a^{\alpha}\right\} \cup g p\left\{b^{\beta}\right\} .
$$

Therefore, (2.1) is reduced. If $n \geq 1$, then it follows from Britton's lemma [5] that equation (2.1) does not define the identity element. When $n=0,(2.1)$ yields $a g_{0} b g_{0}^{-1} a^{-1} g_{0} b^{-1} g_{0}^{-1}$. Then, from [6, Theorem 4.5], this is not the identity element in $H$, and so in $G$. Thus we complete the proof.

LEMMA 2.3. Suppose that $H$ is also described as a tree product $T=\left\{A_{i}, \theta_{j k}\right\}$ of the infinite cyclic groups $A_{i}$. Then, $H$ is a tree product of two vertices, say $A_{1}, A_{2}$, of $\left\{A_{i}\right\}$ and the edge is given by $a_{1}^{p} \rightarrow a_{2}^{q}$, where $a_{i}$ is a generator of $A_{i}, i=1,2$.

PROOF. Since $H$ is finitely generated, we may assume that the number of the vertices $\left\{A_{i}\right\}$ is finite and the tree product $T$ is proper, i.e., each of the amalgamated subgroups is properly contained in the adjoining vertices [4, p. 237]. 
Let $H^{*}$ be the normal closure of the nonextremal vertices of $\left\{A_{i}\right\}$ in $H$. Then, since $T$ is proper, the factor group $H / H^{*}$ is a free product of nontrivial cyclic groups and the rank (i.e., miminum number of generators) of $H / H^{*}$ is equal to the number of extremal vertices of $\left\{A_{i}\right\}$. Since the rank of $H$ is 2 , it follows that $T$ has exactly two extremal vertices (i.e., $T$ is a stem product). Thus, from $[\mathbf{9}$, p. 97 , Theorem 2] and [7, p. 320, Theorem 1], we obtain the lemma.

LEMMA 2.4. The induced presentation $\left\langle a, b, t: a^{p}=b^{q}, t a^{\alpha} t^{-1}=b^{\beta}, t\right\rangle$ of (1.1) is $A C$-equivalent to the trivial presentation $\langle:\rangle$.

PROOF. The lemma is an immediate consequence of [1, Theorem 2.1].

3. Proof of Theorem 1. From Lemma 2.4 and [13], the first assertion holds. We proceed to the proof of the second assertion. We will prove only the case of $p=2, q=3, \alpha=3, \beta=4$, and the other cases can be shown by the same arguments.

Assume that $G$ is an HNN extension of a free group $F$ of finite rank $d$ with a single stable letter $t$. Then the commutator subgroup $G^{\prime}$ of $G$ is the amalgamated free product of infinitely many factors

$$
\cdots * F_{-10} F_{0} * F_{01} F_{1} *_{F_{12}} F_{2} * \cdots,
$$

where $F_{i}$ are copies of $F$. Let $F(i, j)$ denote the subgroup $F_{i} *_{F_{i, i+1}} \cdots * F_{j-1, j} F_{j}, i \leq$ $j$. On the other hand, from $(1.1), G^{\prime}$ is also the amalgamated free product

$$
\cdots *_{H_{-10}} H_{0} *_{H_{01}} H_{1} *_{H_{12}} H_{2} * \cdots,
$$

where $H_{i}=\left\langle a_{i}, b_{i}: a_{i}^{2}=b_{i}^{3}\right\rangle, H_{i, i+1}=g p\left\{a_{i+1}^{3}\right\}=g p\left\{b_{i}^{4}\right\}, a_{i}=t^{i} a t^{-i}$ and $b_{i}=t^{i} b t^{-i}$; each amalgamation is given by mapping $a_{i+1}^{3} \rightarrow b_{i}^{4}$. Let $H(i, j)$ denote the subgroup $H_{i} *_{H_{i, i+1}} \cdots *_{H_{j-1, j}} H_{j}, i \leq j$.

Now, $a_{0}^{2}$ is an element of the commutator subgroup $G^{\prime}$. Hence, by (3.1), there exist integers $r, s(r \leq s)$ such that $a_{0}^{2} \in F(r, s)$. Moreover, since $F(r, s)$ is finitely generated, it follows from (3.2) that there exist integers $n, m(n \leq m)$ such that $F(r, s) \subset H(n, m)$. Here we may assume that $n \leq 0<m$.

The center $C\left(H_{i}\right)$ of $H_{i}$ is the infinite cyclic group generated by $a_{i}^{2}\left(=b_{i}^{3}\right)$. Therefore, from [6, p. 211], we have

$$
\begin{aligned}
C(H(n, n+1)) & =g p\left\{b_{n}^{3}\right\} \cap g p\left\{a_{n+1}^{2}\right\} \cap H_{n, n+1} \\
& =g p\left\{\left(b_{n}^{3}\right)^{8}\right\}=g p\left\{\left(a_{n}^{2}\right)^{8}\right\} .
\end{aligned}
$$

Similarly, we can obtain

$$
C(H(n, m))=g p\left\{\left(a_{n}^{2}\right)^{8^{m-n}}\right\}
$$

and it is infinite cyclic. Since $\left(a_{0}^{2}\right)^{9}=\left(a_{-1}^{2}\right)^{8}$, we can see that

$$
\left\{\left(a_{0}^{2}\right)^{9^{-n}}\right\}^{8^{m}}=\left\{\left(a_{n}^{2}\right)^{8^{-n}}\right\}^{8^{m}}=\left(a_{n}^{2}\right)^{8^{m-n}} \in C(H(n, m)) .
$$

Thus, since $\left\{\left(a_{0}^{2}\right)^{9^{-n}}\right\}^{8^{m}} \in F(r, s) \subset H(n, m)$, it follows that $\left\{\left(a_{0}^{2}\right)^{9^{-n}}\right\}^{8^{m}}(\neq 1)$ is in the center $C(F(r, s))$. Hence $C(F(r, s))$ is nontrivial. Thus, from [6, p. 211], the free group $F_{r}$ must have the nontrivial center. Therefore, we have $d=1$, and $G$ is presented by $\left\langle c, x: x c^{k} x^{-1}=c^{l}\right\rangle$. Now the Alexander polynomial $\Delta(t)$ of $G$ is $9 t-8$. Hence we get $k= \pm 9, l= \pm 8$ or $k= \pm 8, l= \pm 9$. Without loss of generality, we may assume that $k=9, l=8$. 
Thus, if $G$ is an HNN extension of a free group of finite rank, then $G$ must be isomorphic to the group $G^{*}$ presented by

$$
\left\langle c, x: x c^{9} x^{-1}=c^{8}\right\rangle .
$$

To complete the proof, we will show the following:

Claim. $G$ cannot be isomorphic to $G^{*}$.

We assume that there exists an isomorphism $\Phi$ of $G$ onto $G^{*}$. Let $H=g p\{a, b\}$ in $G$. We consider $G^{*}$ as an HNN extension of $F(=\langle c:\rangle)$ with associated subgroups $g p\left\{c^{9}\right\}$ and $g p\left\{c^{8}\right\}$. Using the subgroup theorem for HNN extensions in [3], we can describe the subgroup $\Phi(H)$ of $G^{*}$ as follows:

$\Phi(H)$ is an HNN extension with stable letters $t_{1}, \ldots, t_{n}(n \geq 0)$ whose base is a tree product of vertices $d F d^{-1} \cap \Phi(H)$ where $d$ ranges over a double coset representative system for $G^{*} \bmod (\Phi(H), F)$; the amalgamated and associated subgroups are contained in vertices of this base.

We can see the following:

(1) $d F d^{-1} \cap \Phi(H) \cong 1$ or $Z$.

(2) $n=0$.

The first assertion follows immediately from the fact that $F \cong Z$. Since $H_{1}(\Phi(H))$ $\cong Z$, it follows that $n$ is at most one. If $n=1$, then its associated subgroups must be isomorphic to 1 or $Z$. However, this is impossible for the group $\Phi(H)$. Hence, we conclude that $n=0$.

Thus, $\Phi(H)$ is a tree product of infinite cyclic groups $d F d^{-1} \cap \Phi(H)$. Therefore, from Lemma 2.3, $\Phi(H)$ is an amalgamated product of two vertices $d_{1} F d_{1}^{-1} \cap \Phi(H)$ and $d_{2} F d_{2}^{-1} \cap \Phi(H)$ with subgroups $g p\left\{u_{1}^{2}\right\}$ and $g p\left\{u_{2}^{3}\right\}$ amalgamated under $u_{1}^{2} \rightarrow$ $u_{2}^{3}$, where $u_{i}(i=1,2)$ is a generator of $d_{i} F d_{i}^{-1} \cap \Phi(H)$. Thus, we have

$$
\Phi(H)=\left\langle u_{1}, u_{2}: u_{1}^{2}=u_{2}^{3}\right\rangle .
$$

Hence, there exists an automorphism $f$ of $\Phi(H)$ such that $\Phi(a)=f\left(u_{1}\right)$ and $\Phi(b)=$ $f\left(u_{2}\right)$. From [12], the automorphism $f$ of $\Phi(H)$ is given by the form

$$
f\left(u_{1}\right)=W u_{1}^{\varepsilon} W^{-1}, \quad f\left(u_{2}\right)=W u_{2}^{\varepsilon} W^{-1},
$$

where $W \in \Phi(H)$ and $\varepsilon= \pm 1$. Thus we have

$$
\Phi(a)=W u_{1}^{\varepsilon} W^{-1}, \quad \Phi(b)=W u_{2}^{\varepsilon} W^{-1} .
$$

Now, since $F=\langle c:\rangle$, it follows that $u_{i}=d_{i} c^{\nu_{i}} d_{i}^{-1}, i=1,2$, where $\nu_{i}$ are nonzero integers. Hence we can easily see that

$$
u_{1}\left(d_{1} d_{2}^{-1} u_{2} d_{2} d_{1}^{-1}\right) u_{1}^{-1}=d_{1} d_{2}^{-1} u_{2} d_{2} d_{1}^{-1} \text {. }
$$

Thus, we obtain

$$
\begin{aligned}
1 & =\Phi^{-1}\left(W u_{1} d_{1} d_{2}^{-1} u_{2} d_{2} d_{1}^{-1} u_{1}^{-1} d_{1} d_{2}^{-1} u_{2}^{-1} d_{2} d_{1}^{-1} W^{-1}\right) \\
& =a^{\varepsilon} \Phi^{-1}(S) b^{\varepsilon} \Phi^{-1}\left(S^{-1}\right) a^{-\varepsilon} \Phi^{-1}(S) b^{-\varepsilon} \Phi^{-1}\left(S^{-1}\right),
\end{aligned}
$$

where $S=W d_{1} d_{2}^{-1} W^{-1}$. Therefore, there exists $\tilde{S} \in G$ such that

$$
a\left(\tilde{S} b \tilde{S}^{-1}\right) a^{-1}\left(\tilde{S} b^{-1} \tilde{S}^{-1}\right)=1 .
$$

However, this contradicts Lemma 2.2. Hence, there cannot exist an isomorphism of $G$ onto $G^{*}$. Thus, we complete the proof of the claim, and therefore Theorem 1 . 
REMARKS. (1) It can be shown that if an $n$-knot group $G$ is an HNN extension of a free group of infinite rank, then $G$ has a free base of finite rank (cf. [14]). Therefore, the groups in Theorem 1 have no free base.

(2) Each group in Theorem 1 is a ribbon $n$-knot group $(n \geq 2)$ but not a 1-knot group because its Alexander polynomial has degree one. L. Neuwirth [8] proved that if the commutator subgroup of a 1-knot group is finitely generated, then it is free of finite rank. It still remains open whether there exists a 1-knot group which has no free base.

4. Knot groups with one-relator Wirtinger presentation. In this section, we will show that

THEOREM 2. Any 2-knot group with one-relator Wirtinger presentation has a free base of finite rank.

ProOF. Let $G$ be a group presented by

$$
\left\langle x, y: y=W(x, y) x W(x, y)^{-1}\right\rangle,
$$

where $W(x, y)$ is a reduced word $(\neq 1)$ which does not begin in $y$ or $y^{-1}$ and does not end in $x$ or $x^{-1}$. Setting $a=y x^{-1}$ and deleting $y$ in (4.1), we obtain

$$
\begin{aligned}
G & =\left\langle x, a: a x=W(x, a x) x W(x, a x)^{-1}\right\rangle \\
& =\left\langle x, a: x W^{\prime} x^{-1}=a^{-1} W^{\prime}\right\rangle,
\end{aligned}
$$

where $W^{\prime}$ is the reduced word of $W(x, a x)$. We notice that

$W^{\prime}$ begins in neither $a$ nor $x^{-1} a^{-1}$.

Let $\tilde{W}\left(a_{i}\right)$ be the word obtained by rewriting $W^{\prime} x^{-k}$, where $k$ is the exponent sum of $W^{\prime}$ on $x$, in terms of $a_{i}=x^{i} a x^{-i}$. Let $m$ be the minimum and $M$ the maximum subscript $j$ such that $a_{j}$ occurs in $\tilde{W}\left(a_{i}\right)$. If $M \geq 0$, then we rewrite the relation in (4.2) as follows:

$$
x \tilde{W}\left(a_{i}\right) x^{-1}=a_{0}^{-1} \tilde{W}\left(a_{i}\right) .
$$

If $M<0$, then we have

$$
x\left(a x W^{\prime} x^{-1} W^{\prime-1}\right) x^{-1}=x a_{0} \tilde{W}\left(a_{i+1}\right) x^{-1} \tilde{W}\left(a_{i+1}\right)^{-1}=1 .
$$

Thus, we obtain

$$
G=\left\langle a_{\delta}, a_{\delta+1}, \ldots, a_{D}, x: x a_{\delta} x^{-1}=a_{\delta+1}, \ldots, x a_{D-1} x^{-1}=a_{D}, x P x^{-1}=Q\right\rangle,
$$

where $D=\max \{M, 0\}$, and $\delta, P, Q$ are given by

(1) if $M \geq 0$, then $\delta=\min \{m, 0\}, P=\tilde{W}\left(a_{i}\right), Q=a_{0}^{-1} \tilde{W}\left(a_{i}\right)$,

(2) if $M<0$, then $\delta=m+1, P=a_{0} \tilde{W}\left(a_{i+1}\right), Q=\tilde{W}\left(a_{i+1}\right)$.

From $(*), \tilde{W}\left(a_{i}\right)$ does not begin in $a_{0}$, and $\tilde{W}\left(a_{i+1}\right)$ does not begin in $a_{0}^{-1}$. Therefore, in both cases of (1), (2), $P$ involves $a_{D}$ and $Q$ involves $a_{\delta}$. Consequently, $\left\{a_{\delta}, \ldots, a_{D-1}, P\right\}$ and $\left\{a_{\delta+1}, \ldots, a_{D}, Q\right\}$ freely generate free subgroups of rank $D-\delta+1$ in the free group $F$ on $a_{\delta}, \ldots, a_{D}$, respectively. It follows that $F$ is a free base of $G$. Thus we obtain the theorem.

REMARKS. (1) There exists a "one-relator" 2-knot group which has no free base. For example, in the case of $p=2, q=3, \alpha=3, \beta=4$, the group given by (1.1) is a one-relator group.

(2) In [11, p. 125], E. S. Rapaport showed that for any one-relator 2-knot group $G$, its commutator subgroup $G^{\prime}$ is finitely generated if and only if $G^{\prime}$ is free. 


\section{REFERENCES}

1. J. Andrews and M. Curtis, Extended Nielsen operations in free groups, Amer. Math. Monthly 73 (1966), 21-28.

2. M. A. Gutierrez, On the Seifert manifold of a 2-knot, Trans. Amer. Math. Soc. 240 (1978), 287-294.

3. A. Karrass, A. Pietrowski and D. Solitar, An improved subgroup theorem for HNN groups with some applications, Canad. J. Math. 26 (1974), 214-224.

4. A. Karrass and D. Solitar, The subgroups of a free product of two groups with an amalgamated subgroup, Trans. Amer. Math. Soc. 150 (1970), 227-255.

5. R. C. Lyndon and P. Schupp, Combinatorial group theory, Springer-Verlag, Berlin and New York, 1977.

6. W. Magnus, A. Karrass and D. Solitar, Combinatorial group theory, Pure and Appl. Math., vol. 13, Interscience, New York, 1966.

7. S. Meskin, A. Pietrowski and A. Steinberg, One-relator groups with center, J. Austral. Math. Soc. 16 (1973), 319-323.

8. L. Neuwirth, The algebraic determination of the groups of knots, Amer. J. Math. 82 (1960), 791-798.

9. A. Pietrowski, The isomorphism problem for one-relator groups with non-trivial centre, Math. Z. 136 (1974), 95-106.

10. E. S. Rapaport, Remarks on groups of order 1, Amer. Math. Monthly 75 (1968), 714-720.

11. __ Knot-like groups, Knots, Groups and 3-Manifolds, Ann. of Math. Studies, no. 88, Princeton Univ. Press, Princeton, N.J., 1975, pp. 119-133.

12. O. Schreier, Über die Gruppen $A^{a} B^{b}=1$, Abh. Math. Sem. Univ. Hamburg 3 (1923), 167-169.

13. K. Yoshikawa, $A$ note on Levine's conditions for knot groups, Math. Sem. Notes Kobe Univ. 10 (1982), 633-636.

14. __ On n-knot groups which have abelian bases, preprint.

Faculty of Science, KWansei Gakuin University, Nishinomiya, Hyogo 662, JAPAN 